\title{
Outcome of patients with relapsed or refractory acute myeloid leukemia treated with Mito-FLAG salvage chemotherapy
}

\author{
Regina Mühleck ${ }^{1}$. Sebastian Scholl ${ }^{1}$. Inken Hilgendorf ${ }^{1} \cdot$ Karin Schrenk $^{1}$. Jakob Hammersen ${ }^{1}$. Jochen J. Frietsch ${ }^{1}$. \\ Maximilian Fleischmann ${ }^{1} \cdot$ Herbert G. Sayer ${ }^{2} \cdot$ Anita Glaser $^{3}$. Andreas Hochhaus ${ }^{1}$. Ulf Schnetzke ${ }^{1}$ (1)
}

Received: 20 August 2021 / Accepted: 25 September 2021 / Published online: 5 October 2021

(c) The Author(s) 2021

\begin{abstract}
Purpose Curative intended treatment is challenging in patients with relapsed or refractory acute myeloid leukemia (r/r AML) and associated with a dismal prognosis for long-term survival. Despite novel treatment options, the majority of patients are treated with chemotherapy-based regimens. Although widely used, little data exist on the combination of fludarabine, cytarabine, granulocyte colony stimulating factor (FLAG) and mitoxantrone as salvage strategy for $\mathrm{r} / \mathrm{r}$ AML.

Materials and methods Sixty-six patients receiving Mito-FLAG for $\mathrm{r} / \mathrm{r}$ AML treated at a German tertiary care center between 2009 and 2019 were analyzed with regard to response rates, survival and safety profile.

Results Overall response rate was $75.8 \%$ with $56.1 \%$ of patients achieving complete remission (CR) and $19.7 \%$ partial remission (PR). After a median follow-up of 54 months, median overall survival (OS) was 13 months. Patients transitioned to allogeneic hematopoietic stem cell transplantation (alloHSCT) $(75.8 \%)$ showed a significant improvement in OS with a median OS of 17 (95\% CI 8.5-25.4) months vs 3 (95\% CI 1.7-4.3) months $(p<0.001)$. 30- and 60-day mortality rates for all patients after the initial cycle of Mito-FLAG were $4.5 \%$ and $7.6 \%$, respectively.

Conclusion The Mito-FLAG salvage protocol represents an effective and feasible treatment regimen for $\mathrm{r} / \mathrm{r}$ AML. Importantly, a high rate of transition to successful alloHSCT with the aim of long-term disease-free survival has been shown.
\end{abstract}

Keywords AML $\cdot$ Refractory $\cdot$ Relapse $\cdot$ Salvage therapy $\cdot$ Mito-FLAG

\section{Introduction}

Effective salvage therapies present an urgent medical need in patients with relapsed or refractory acute myeloid leukemia ( $\mathrm{r} / \mathrm{r}$ AML) (Döhner et al. 2017; Thol et al. 2015). Intensive induction chemotherapy and consolidation by conventional chemotherapy and/or allogeneic hematopoietic stem cell transplantation (alloHSCT) remain the cornerstone of curative intended AML treatment. $60-80 \%$ of younger patients achieve complete remission (CR) following induction

Ulf Schnetzke

ulf.schnetzke@med.uni-jena.de

1 Klinik für Innere Medizin II, Abteilung für Hämatologie und Internistische Onkologie, Universitätsklinikum Jena, Am Klinikum 1, 07747 Jena, Germany

2 4. Medizinische Klinik, HELIOS Klinikum Erfurt, Nordhäuser Straße 74, 99089 Erfurt, Germany

3 Institut für Humangenetik, Universitätsklinikum Jena, Am Klinikum 1, 07747 Jena, Germany chemotherapy. About $50 \%$ of patients older than 60 years treated with induction chemotherapy respond adequately (Döhner et al. 2015; Dombret and Gardin 2016).

In contrast, a considerable proportion of patients subsequently relapse and need further treatment. Hence, only about $30-50 \%$ of patients younger than 60 years and $10-20 \%$ of patients older than 60 years are cured from the disease (Röllig et al. 2020; Short et al. 2018). Recently, new therapeutic agents were implemented into classical "7 plus 3" induction chemotherapy and salvage regimens (Kantarjian et al. 2021; Heuser et al. 2020).

Distinct cytogenetically and molecularly defined entities in AML are crucial in terms of treatment and prognosis of the disease (Döhner et al. 2017; Estey 2020). The heterogenous group of the AML landscape requires different selective therapeutic options.

To date, curative intended $\mathrm{r} / \mathrm{r}$ AML is treated with intensified (re-) induction protocols, typically comprising high-dose cytarabine as a backbone and different anthracycline and alkylating counterparts (Burnett et al. 2013). A 
direct comparison of those salvage regimens has not been performed so that a standard regime has not been defined. Commonly applied treatment protocols are fludarabine, cytarabine, granulocyte colony stimulating factor, and idarubicin (FLAG-Ida) and mitoxantrone, etoposide, cytarabine (MEC) (Thol et al. 2015). Rates of achieving CR including CR with incomplete hematologic recovery (CRi) are about 50\% (Steinmetz et al. 1999; Westhus et al. 2019; Parker et al. 1997; Spadea et al. 1993). Long-term survival is still dismal with cure rates between 10 and $40 \%$ which depend on the option of subsequent alloHCST (Forman and Rowe 2013; Kell 2006; Schmid et al. 2006). A modified FLAG-protocol containing mitoxantrone (Mito-FLAG) has been shown to be effective as a salvage regimen for $\mathrm{r} / \mathrm{r}$ AML in a multicenter, randomized phase 3 trial comparing cytarabine as bolus versus continuous infusion (Thiel et al. 2015; Hänel et al. 2001). Here, we present efficacy data of Mito-FLAG as a potent chemotherapy protocol even for high-risk patients according to the European LeukemiaNet (ELN) guidelines. In addition, we compare those data with a previous salvage chemotherapy protocol containing high-dose cytarabine and cyclophosphamide (hAC) (Schnetzke et al. 2014).

\section{Patients and methods}

\section{Patients}

Patients with r/r AML (excluding AML M3) who received Mito-FLAG either due to refractory or relapsed disease were analyzed. All patients were treated at University Hospital Jena, Germany, between 2009 and 2019 and included in one of the following AML registries: AML registry of the OSHO study group (East German Study Group of Hematology and Oncology) or in the SAL registry (Study Alliance Leukemia). Patients gave their written consent for data acquisition and analysis after pseudonymization in one of the registries. This retrospective data analysis was approved by the local ethics committee of the University Hospital Jena, Germany (Ethical numbers 4871-07/16 for "retrospective evaluation of therapy response and survival in patients with AML" and 3967-12/13 for SAL registry).

\section{Mito-FLAG treatment protocol}

Mito-FLAG protocol consisted of fludarabine $\left(15 \mathrm{mg} / \mathrm{m}^{2} /\right.$ bid, $15 \mathrm{~min}$ infusion) and cytarabine $\left(1 \mathrm{~g} / \mathrm{m}^{2} / \mathrm{bid}, 3 \mathrm{~h}\right.$ infusion) for 5 consecutive days. In addition, mitoxantrone ( $7 \mathrm{mg} / \mathrm{m}^{2}, 30 \mathrm{~min}$ infusion) was administered on days 1 , 3 , and 5. Granulocyte colony stimulating factor (G-CSF) was initiated subcutaneously on day 0 until hematologic recovery.
The hAC-regimen was administered as published previously (Schnetzke et al. 2014). Briefly, cytarabine $\left(3 \mathrm{~g} / \mathrm{m}^{2} /\right.$ bid) ( $3 \mathrm{~h}$ infusion) at days $1-4$ and cyclophosphamide ( $1 \mathrm{~g} /$ $\mathrm{m}^{2}$ ) on days 1 and 3 were applied.

\section{Treatment administration}

Induction and consolidation chemotherapy were applied either according to the OSHO 2002, OSHO 2004 or " $7+3$ " protocol. In younger patients ( $\leq 60$ years), OSHO protocol consisted of idarubicin $\left(12 \mathrm{mg} / \mathrm{m}^{2}\right.$, days $\left.1-3\right)$ and intermediate-dosed cytarabine $\left(1 \mathrm{~g} / \mathrm{m}^{2} / \mathrm{bid}\right.$, days $1,3,5$ and 7$)$ as induction chemotherapy, while patients over 60 years received mitoxantrone $\left(10 \mathrm{mg} / \mathrm{m}^{2}\right.$, days $\left.1-3\right)$ and intermediate-dosed cytarabine $\left(1 \mathrm{~g} / \mathrm{m}^{2} / \mathrm{bid}\right.$, days $1,3,5$ and 7$)$ as induction treatment. First consolidation chemotherapy in younger AML patients was identical with induction chemotherapy. Elderly AML patients underwent consolidation treatment with a dose reduction of mitoxantrone $(10 \mathrm{mg} /$ $\mathrm{m}^{2}$, days 1 and 2) and intermediate-dosed cytarabine ( $0.5 \mathrm{~g} /$ $\mathrm{m}^{2} /$ bid, days 1, 3 and 5) (Büchner et al. 2012; Kahl et al. 2016). Standard " $7+3$ " induction regimen was applied as previously published with a continuous 7-day infusion of cytarabine $\left(100 \mathrm{mg} / \mathrm{m}^{2} /\right.$ day $)$ and daunorubicin $\left(45 \mathrm{mg} / \mathrm{m}^{2}\right.$, days 1-3) (Wiernik et al. 1992). For consolidation therapy following " $7+3$ ", high-dose cytarabine $\left(3 \mathrm{~g} / \mathrm{m}^{2} / \mathrm{bid}\right)$ for 3 days (day $1,3,5$ ) was applied.

AlloHSCT was performed whenever feasible due to the $\mathrm{r} / \mathrm{r}$ AML as high-risk disease per se.

The majority of patients subsequently undergoing alloHSCT received a reduced-toxicity conditioning (RTC) based on treosulfan or busulfan in combination with fludarabine with or without ATG prior to transplantation $(n=42$, 84\%) (Casper et al. 2012; Kröger et al. 2003). All remaining patients underwent myeloablative conditioning (MAC) $(n=8,16 \%)$ (Jethava et al. 2017). The characteristics of patients who underwent alloHSCT and donor type distribution are summarized in Table S1.

\section{Response evaluation}

Overall response rate was defined as complete remission (CR), complete remission with incomplete recovery (CRi) and partial remission (PR). Additional efficacy assessments were performed by calculation for overall survival (OS) and event-free survival (EFS). OS is defined as date of initiation of Mito-FLAG to the date of death from any cause. EFS is the date of initiation of Mito-FLAG to death for any reason, refractory or relapse disease. Response criteria were used according to the ELN 2017 guidelines (Döhner et al. 2017).

Following Mito-FLAG application, first response evaluation was on day 15 . Here, a blast count of $5 \%$ or less in the bone marrow was assessed as clearance of blasts. Remission 
evaluation was usually performed after hematologic recovery. $\mathrm{CR}$ was defined as $5 \%$ blasts or less within the bone marrow and adequate peripheral blood counts (neutrophils $\geq 1.0 \times 10^{9} / \mathrm{l}$, platelets $\geq 100 \times 10^{9} / 1$ ), while patients with CRi did not show peripheral hematologic recovery. Partial remission was defined as $5-25 \%$ blasts in the bone marrow and a total reduction of blasts of at least $50 \%$ of AML blasts. Refractory disease comprises $>25 \%$ blasts in the bone marrow or less than $50 \%$ reduction defined as persistence of blasts (BP). Failure to attain blast clearance or CR following first-line induction chemotherapy was defined as (primary) refractory disease.

\section{Safety analyses}

Non-hematologic toxicity of Mito-FLAG regimen was evaluated according to the Common Terminology Criteria and Adverse Events (CTCAE v5.0). Evaluation of the hematologic toxicity was performed by analysis of duration of neutrophils below $0.5 \times 10^{9} / 1$ subsequent to Mito-FLAG application.

\section{Genetic and molecular analysis}

Cytogenetic evaluation was performed with standard banding techniques, and karyotypes were described according to the currently valid International System for Human Cytogenetic Nomenclature (McGowan-Jordan et al. 2021). Cytogenetic categorization into favorable, intermediate or adverse risk was performed on the basis of recommended criteria (Döhner et al. 2017).

The presence of FLT3-ITD and NPM1 mutations was detected by PCR amplification of the corresponding region using genomic DNA followed by fragment analysis (Scholl et al. 2005, 2008).

\section{Statistical considerations}

Binary logistic regression model was conducted to assess the impact of independent variables.

Time-to-event analyses (OS, EFS) were estimated using the Kaplan-Meier method and compared using log-rank test.

$\mathrm{Chi}^{2}$-test and Fisher's exact test were applied for comparing response rates of the Mito-FLAG cohort to the hAC cohort ( $n=22$ patients), whereas survival data were analyzed by logrank test, respectively. $p$ values below 0.05 were considered as statistically significant. Statistical analyses were performed by IBM SPPS v24.0 (SPSS, Chicago, IL, USA).

\section{Results}

\section{Demographics}

Sixty-six patients (female $51.5 \%$, median age at MitoFLAG initiation 56 years; range 21-71 years) were analyzed for this study (Table 1). All patients retained a good performance status with an ECOG of 2 or less. 34 patients had de novo AML, 23 secondary AML (sAML), and 6 treatment-related AML (tAML). FLT3-ITD diagnostics were performed in 60 patients with 16 of 60 (27\%) harboring an activating mutation. Cytogenetics could be carried out in 65 of 66 patients with $6(9 \%), 38(58 \%)$ and $21(32 \%)$ considered as low, intermediate and high risk according to cytogenetic risk classification (Table 1).

\section{Treatment characteristics and disease status prior to Mito-FLAG}

Prior to Mito-FLAG salvage regimen all patients received cytarabine-based induction therapy with 54 (82\%) patients according to OSHO protocols and $10(15 \%)$ " $7+3$ " regimen. Of the two remaining patients, one received all-trans retinoic acid, idarubicin and cytarabine, whereas the other was treated with CPX-351 as induction therapy.

Prior to Mito-FLAG therapy 30 patients (45\%) received at least one cycle of consolidation therapy (Table 1).

Most $\mathrm{r} / \mathrm{r}$ AML patients were in first salvage with 36 (54.5\%) patients who did not achieve CR following induction therapy and $30(45.5 \%)$ patients who received MitoFLAG due to relapse. Except for one patient, Mito-FLAG was applied for first relapse. Of note, most of relapsing patients presented with early relapse, defined as complete remission for 12 months or less $(28.8 \%$ early relapse vs $16.7 \%$ late relapse) as summarized in Table 1.

\section{Adverse events}

Grade 3 and 4 adverse events (AEs) according to CTCAE occurring in $\geq 10 \%$ of patients included febrile neutropenia (86.4\%), mucositis (22.7\%) and elevated liver enzymes (15.2\%) (Table 2). The most common site of infection was pneumonia $(48.5 \%)$ followed by central line infections.

30-day and 60-day mortality after the initial cycle of Mito-FLAG was $4.5 \%$ and $7.6 \%$ ( 3 and 5 patients), respectively. Four patients died of infectious complications, and one patient had a fatal outcome due to liver failure. Subsequent to a second cycle of Mito-FLAG, three more patients died of severe sepsis. 
Table 1 Patient demographics

\begin{tabular}{|c|c|}
\hline & $n=66$ \\
\hline Median age at Mito-FLAG, years (range) & $56(21-71)$ \\
\hline Sex, female $(\%)$ & $34(51.5)$ \\
\hline \multicolumn{2}{|l|}{ ECOG-performance status, n (\%) } \\
\hline $0-1$ & $58(87.9)$ \\
\hline 2 & $5(7.6)$ \\
\hline Unknown & $3(4.5)$ \\
\hline \multicolumn{2}{|l|}{ AML type, $n(\%)$} \\
\hline De novo & $34(51.5)$ \\
\hline sAML & $23(34.9)$ \\
\hline tAML & $6(9.1)$ \\
\hline Unknown & $3(4.5)$ \\
\hline \multicolumn{2}{|l|}{ Molecular genetics, $n(\%)$} \\
\hline NPM1 mutation & $10(15.2)$ \\
\hline NPM 1 wild type & $32(48.5)$ \\
\hline Unknown & $24(36.3)$ \\
\hline FLT3-ITD mutation & $16(24.2)$ \\
\hline FLT3 wild type & $44(66.7)$ \\
\hline Unknown & $6(9.1)$ \\
\hline \multicolumn{2}{|l|}{ Cytogenetic prognostic group, $n(\%)$} \\
\hline Favorable & $6(9.1)$ \\
\hline Intermediate & $38(57.6)$ \\
\hline Adverse & $21(31.8)$ \\
\hline Unknown & $1(1.5)$ \\
\hline \multicolumn{2}{|l|}{ ELN-risk group } \\
\hline Favorable & $10(15.2)$ \\
\hline Intermediate & $18(27.3)$ \\
\hline Adverse & $24(36.3)$ \\
\hline Unknown & $14(21.2)$ \\
\hline \multicolumn{2}{|l|}{ FAB-classification, $n(\%)$} \\
\hline M0 & $5(7.6)$ \\
\hline $\mathrm{M} 1 / 2$ & $30(45.4)$ \\
\hline M4/5 & $25(37.9)$ \\
\hline M6 & $1(1.5)$ \\
\hline M7 & $1(1.5)$ \\
\hline Unknown & $4(6.1)$ \\
\hline \multicolumn{2}{|l|}{ Induction therapy prior to Mito-FLAG, $n(\%)$} \\
\hline OSHO $\leq 60$ years & $42(63.6)$ \\
\hline OSHO $>60$ years & $12(18.2)$ \\
\hline$" 7+3 "$ & $10(15.2)$ \\
\hline Other & $2(3)$ \\
\hline \multicolumn{2}{|l|}{ Consolidation therapy prior to Mito-FLAG, $n(\%)$} \\
\hline None & $36(54.6)$ \\
\hline $\mathrm{OSHO} \leq 60$ years & $24(36.4)$ \\
\hline OSHO $>60$ years & $3(4.5)$ \\
\hline HD-cytarabine & $3(4.5)$ \\
\hline \multicolumn{2}{|l|}{ Disease status at Mito-FLAG, $n(\%)$} \\
\hline Primary refractory/No CR & $36(54.5)$ \\
\hline PR after induction & $15(22.7)$ \\
\hline $\mathrm{BP}$ after induction & $5(7.6)$ \\
\hline No blast clearance @ day 15 of induction & $16(24.2)$ \\
\hline
\end{tabular}

Table 1 (continued)

\begin{tabular}{ll}
\hline & $n=66$ \\
\hline Relapse & $30(45.5)$ \\
Early relapse & $19(28.8)$ \\
Late relapse & $11(16.7)$ \\
First relapse & 29 \\
Second relapse & 1 \\
Relapse after alloHSCT & 8 \\
\hline
\end{tabular}

Mito-FLAG: mitoxantrone fludarabine cytarabine granulocyte colony stimulating factor; ECOG: Eastern Cooperative Oncology Group; AML: acute myeloid leukemia; sAML: secondary AML; tAML: therapy-related AML; NPM1: nucleophosmin 1; FLT3-ITD: Fms like tyrosinkinase 3-Internal tandem duplication; ELN: European Leukemia Net; FAB: French-American-British Group; LDH: lactate dehydrogenase; OSHO: Ostdeutsche Studiengruppe Hämatologie und Onkologie; HD-cytarabine: high-dose cytarabine; CR: complete response; PR: partial response; BP: blast persistence; alloHSCT: allogeneic hematopoietic stem cell transplantation; early relapse: relapse within $\leq 12$ months; late relapse: relapse after $>12$ months CR

\section{Efficacy}

Figure 1 provides a CONSORT diagram of the Mito-FLAG cohort. CR including CRi (CR with incomplete count recovery) was documented in 37 out of 66 patients $(56.1 \%)$ and PR in 13 patients (19.7\%) (Fig. 1). Six patients (9.1\%) demonstrated no reduction in blast count defined as persistence of blasts (BP). Three patients showed an aplastic bone marrow, whereas one out of three underwent alloHSCT and achieved CR subsequently. Several variables were tested by binary logistic regression analysis for correlation achieving CR (Fig. 2) with disease status (refractory or relapse) at the time of Mito-FLAG application being the only variable that was statistically significant. In detail, patients that were treated for refractory disease showed a greater likelihood of achieving CR than relapsed patients (OR 4.49, 95\% CI $1.59-12.72, p=0.003)$. Of note, refractory disease before Mito-FLAG salvage included patients that did not attain CR following induction chemotherapy and patients who did not show blast clearance at day 15 of induction therapy.

For consolidation, a second Mito-FLAG was applied in 20 out of 66 patients (30.3\%). OS and EFS were calculated for the whole Mito-FLAG cohort with a median follow-up of 54 months (Fig. 3). OS at 1, 3 and 5 years was $54.2 \%, 30 \%$ and $25 \%$, respectively. Median OS for the whole cohort of $\mathrm{r} / \mathrm{r}$ AML patients was 13 (95\% CI 10.2-15.8) months (Fig. 3A).

An event (death of any reason, refractory or relapse disease) was noted in 48 patients (72.7\%). Median EFS was 9 (95\% CI 5.1-12.9) months and probability of 1-, 3- and 5-year EFS was 38.9\%, 26\% and 24\%, respectively (Fig. 3B).

Depending on the response to Mito-FLAG therapy, the median OS was 17 (95\% CI 8.6-25.4) months if a CR/ CRi, 13 (95\% CI 8.3-17.7) months if PR was achieved 
Table 2 Adverse events, $n(\%)$

\begin{tabular}{llllll}
\hline Adverse event & CTCAE-grade 1/2 & CTCAE-grade 3/4 & $\begin{array}{l}\text { CTCAE- } \\
\text { grade 5 } \\
\text { (death) }\end{array}$ & Total & ND \\
\hline Febrile neutropenia/infection & $2(3)$ & $57(86.4)$ & $4(6.1)$ & $63(95.5)$ & $2(3)$ \\
Diarrhea & $30(45.5)$ & $6(9.1)$ & 0 & $36(54.6)$ & $6(9.1)$ \\
Vomiting & $27(40.9)$ & $5(7.6)$ & 0 & $32(48.5)$ & $9(13.6)$ \\
Mucositis & $17(25.8)$ & $15(22.7)$ & 0 & $32(48.5)$ & $9(13.6)$ \\
Cardiac & $23(34.8)$ & $6(9.1)$ & 0 & $29(43.9)$ & $7(10.6)$ \\
Elevation of liver enzymes & $40(60.6)$ & $10(15.2)$ & $1(1.5)$ & $51(77.3)$ & $6(9.1)$ \\
Elevation of creatinine/renal & $11(16.7)$ & $2(3)$ & 0 & $13(19.7)$ & $5(7.6)$ \\
Skin reaction & $14(21.2)$ & $1(1.5)$ & 0 & $15(22.7)$ & $10(15.2)$ \\
Myalgia & $3(4.5)$ & 0 & 0 & $3(4.5)$ & $10(15.2)$ \\
Neurological events & 0 & $4(6.1)$ & 0 & $4(6.1)$ & $9(13.6)$ \\
\hline
\end{tabular}

CTCAE: Common Terminology Criteria for Adverse Events; ND: no data

Fig. 1 CONSORT diagram, antileukemic responses

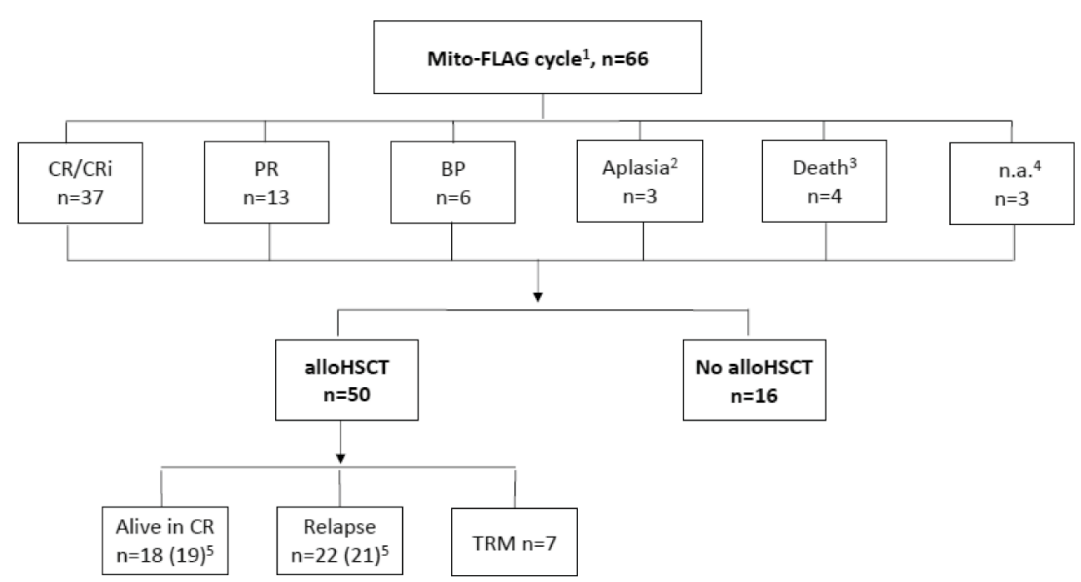

${ }^{1}$ One cycle Mito-FLAG, $n=66$ patients; 2 cycles Mito-FLAG, $n=20$ patients

${ }^{2} 1$ patient with AlloHSCT, 1 with PR and 1 with BP subsequently

${ }^{3}$ death without response assessment

${ }^{4} 1$ patient with blast clearance on day 15 and subsequent alloHSCT; 2 patients with BP on day 15

${ }_{5} 1$ patient recieved second alloHSCT following relapse attaining ongoing CR subsequently

Abbreviations: Mito-FLAG, mitoxantron-fludarabine cytarabin granulocyte-colony stimulating factor; $\mathrm{CR}$, complete response; $\mathrm{CR}(\mathrm{i})$, complete remission with incomplete count recovery; $\mathrm{PR}$, partial response; $\mathrm{BP}$, blast persistence; alloHSCT, allogeneic hematopoietic stem-cell transplantation; n.a., not available; TRM, transplant related mortality and 9 (95\% CI 0-18.6) months if BP was noted (Fig. 3C). The 1-year probability for OS after a CR/CRi was $70.2 \%$, after PR 53.8\% and after BP 33.3\%. The 3- and 5-year probability for CR/CRi was $35 \%$ and $32.2 \%$ and for PR $38.5 \%$ and $23.1 \%$. For OS, a significant difference was noted between patients achieving a CR/CRi, PR or BP following Mito-FLAG $(p=0.01)$.

At data cutoff, 19 out of 66 patients (28.8\%) were in $\mathrm{CR}$ and alive. All of them received an alloHSCT.

\section{Role of alloHSCT}

Following Mito-FLAG, a total of 50 patients $(75.8 \%)$ transitioned to alloHSCT including 34 patients in CR/CRi (see CONSORT diagram, Fig. 1). Treatment characteristics of alloHSCT patients are demonstrated in Table S1. Of note, 42 of 50 patients $(84 \%)$ were conditioned by reduced-toxicity/intensity (RTC/RIC) protocols and 8 patients (16\%) received myeloablative regimens (MAC). Transplant-related 
Fig. 2 Impact of patient/disease characteristics on achieving complete remission following Mito-FLAG. Impact of different independent variables on achieving complete remission calculated by logistic regression

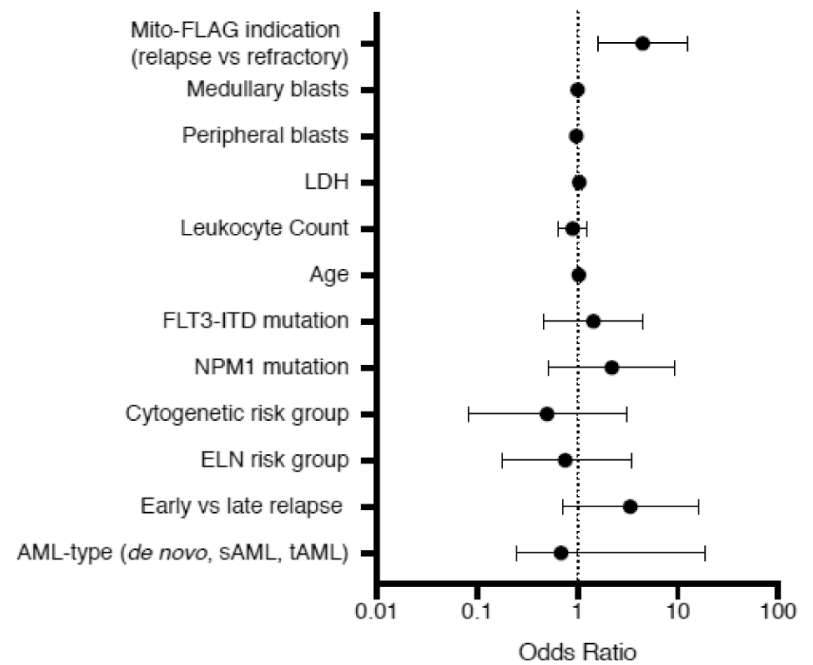

Abbreviations: AML, acute myeloid leukemia; SAML, secondary AML; tAML, therapy related AML; ELN, European Leukemia Net; NPM1, Nucleophosmin-1; FLT3-ITD, Fms like Tyrosinkinase 3-Internal tandem duplication; LDH, lactate dehydrogenase mortality (TRM) was 14\% (7 patients) with infectious complications (5 patients) and graft-versus-host-disease (GvHD) ( 2 patients) as reason of death. At the time of data cutoff $19 / 50$ patients (38\%) following alloHSCT were in CR.

Median OS for patients undergoing alloHSCT was 17 (95\% CI 8.6-25.4) months (Fig. 3D). Of note, patients transplanted in CR had a median OS of 17 (95\% CI 8.03-25.97) months, whereas patients not in CR of 14 (95\% CI 0.28-27.72) months (data not shown, n.s.). The probability of surviving 1 year was $75.9 \%$ for patients in CR and $56.3 \%$ for patients who underwent alloHSCT not in CR (data not shown; n.s.).

Without subsequent alloHSCT, the outcome was significantly worse with a median OS of 3 months (95\% CI $1.7-4.3$ months, $p<0.001)$. 1-year probability of surviving was $69.7 \%$ for patients undergoing alloHSCT and $6.3 \%$ for patients who did not undergo alloHSCT. Of note, there was no difference in age between both groups (alloHSCT vs non-alloHSCT).

\section{Comparison of two different salvage regimens: hAC (high-dose cytarabine and cyclophosphamide) versus Mito-FLAG}

A historical cohort of 22 patients who received high-dose cytarabine ( $3 \mathrm{~g} / \mathrm{m}^{2}, \mathrm{q} 12 \mathrm{~h}$, days $\left.1-4\right)$ and cyclophosphamide $\left(1 \mathrm{~g} / \mathrm{m}^{2}\right)$ days 1 and 3 between 2000 and 2013 was compared to 62 patients of the Mito-FLAG cohort regarding efficacy. Patients who received both protocols were not considered (one patient of the hAC- and 4 patients of the Mito-FLAG cohort).

Patient characteristics for both groups are presented in Table S2. Importantly, the median age was 57 years for the
Mito-FLAG group and 40 years for the hAC group. Indication for salvage therapy was comparable with $50 \%$ (11 patients) refractory and 50\% relapsed AML patients in the hAC group. Overall response rates were comparable for achieving CR/CRi and PR (Table S3). Interestingly, OS and EFS were comparable with respect to different treatment cohorts (Fig. 4A, B).

\section{Discussion}

Despite achieving high CR rates by induction chemotherapy prognosis of $\mathrm{r} / \mathrm{r}$ AML remains unsatisfying (Thiel et al. 2015; Burnett et al. 2015; Fernandez et al. 2009; MegíasVericat et al. 2018). Curative intended treatment for $\mathrm{r} / \mathrm{r}$ AML consists of salvage chemotherapy strategies, typically containing a high-dose cytarabine backbone, e.g., FLAG-Ida or MEC regimen (Döhner et al. 2017; Thol et al. 2015). For distinct subgroups, targeted agents have been approved such as gilteritinib in FLT3-mutated patients (Perl et al. 2019). A direct comparison between conventional intensive chemotherapy-based protocols has not been performed. Therefore, salvage regimens mainly depend on physician's choice and patient's characteristics. Subsequently, alloHSCT has been identified to be the only option of consolidation treatment with the potential to cure r/r AML (Thol et al. 2015).

The Mito-FLAG protocol represents a standard salvage chemotherapy regimen for $\mathrm{r} / \mathrm{r}$ AML, but only little data on efficacy exist (Thiel et al. 2015). Here, a detailed analysis of 66 adult $\mathrm{r} / \mathrm{r}$ AML patients treated at a German tertiary care center receiving Mito-FLAG is presented with regard to safety and outcome. A particular focus was set on patients undergoing alloHSCT. Without this type of consolidation 

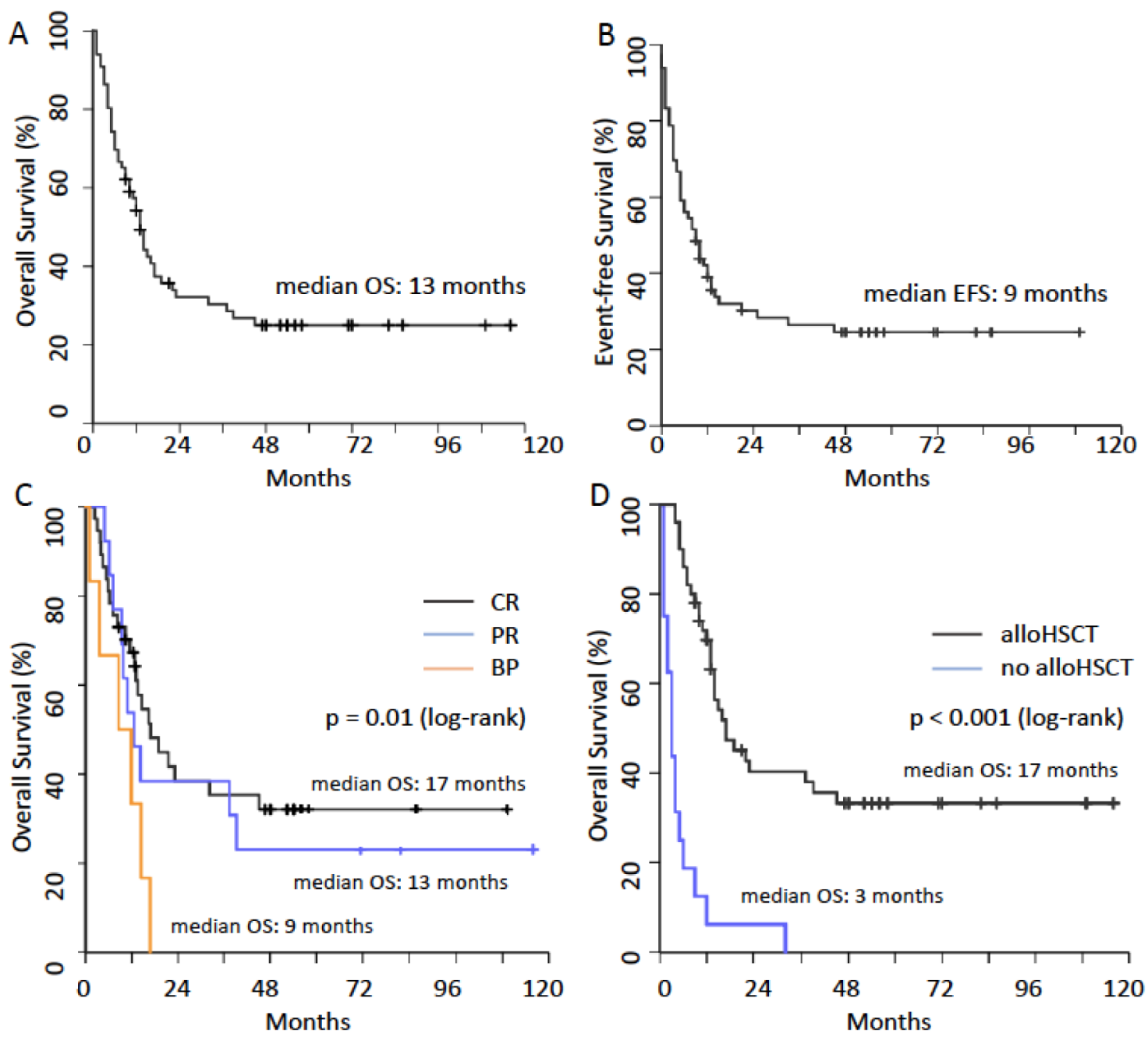

(A) OS, (B) EFS, (C) OS subdivided by remission state, (D) OS depending on alloHSCT

Abbreviations: OS, overall survival; EFS, event-free survival; $C R$, complete response; $P R$, partial response; $B P$, blast persistence; alloHSCT, allogeneic hematopoietic stem-cell transplantation;

Fig. 3 OS and EFS of the whole cohort (A-C) or alloHSCT patients (D) showing months from start of Mito-FLAG

treatment, remission following salvage regimens happens to be only short term (Heuser et al. 2020; Megías-Vericat et al. 2018).

Here, Mito-FLAG salvage treatment resulted in an ORR of $75.8 \%$ including $56.1 \%$ patients in CR/CRi and $19.7 \%$ in $\mathrm{PR}$, respectively. A multicenter, randomized phase III trial comparing cytarabine as bolus versus continuous infusion in $\mathrm{r} / \mathrm{r}$ AML patients represents the most extensive work on Mito-FLAG to date (Heuser et al. 2020; Megías-Vericat et al. 2018). Of 252 patients, 128 were treated with cytarabine bolus application, and a CR rate of $54 \%$ and PR of $15.7 \%$, respectively, was documented which is in accordance to ORR of the Mito-FLAG analysis presented here. A second study published on Mito-FLAG comprised 45 patients with 47\% achieving CR following Mito-FLAG (Luo et al. 2013). Of note, refractory patients were not included in that study and no information on genetics or type of AML was provided.

In general, responses in $\mathrm{r} / \mathrm{r}$ AML vary greatly (CR rates from 20 to $60 \%$ ) by treatment selection, line of therapy and patient characteristics (Döhner et al. 2017; Roboz et al. 2014). A meta-analysis exploring different salvage regimens did reveal CR rates of 44-66\% (Megías-Vericat et al. 2018). The combination of high-dose cytarabine and cyclophosphamide (hAC) was shown to be another potent salvage protocol (Schnetzke et al. 2014).

For this study we compared the response rates and outcome of $22 \mathrm{hAC}$ patients and 62 Mito-FLAG patients who 

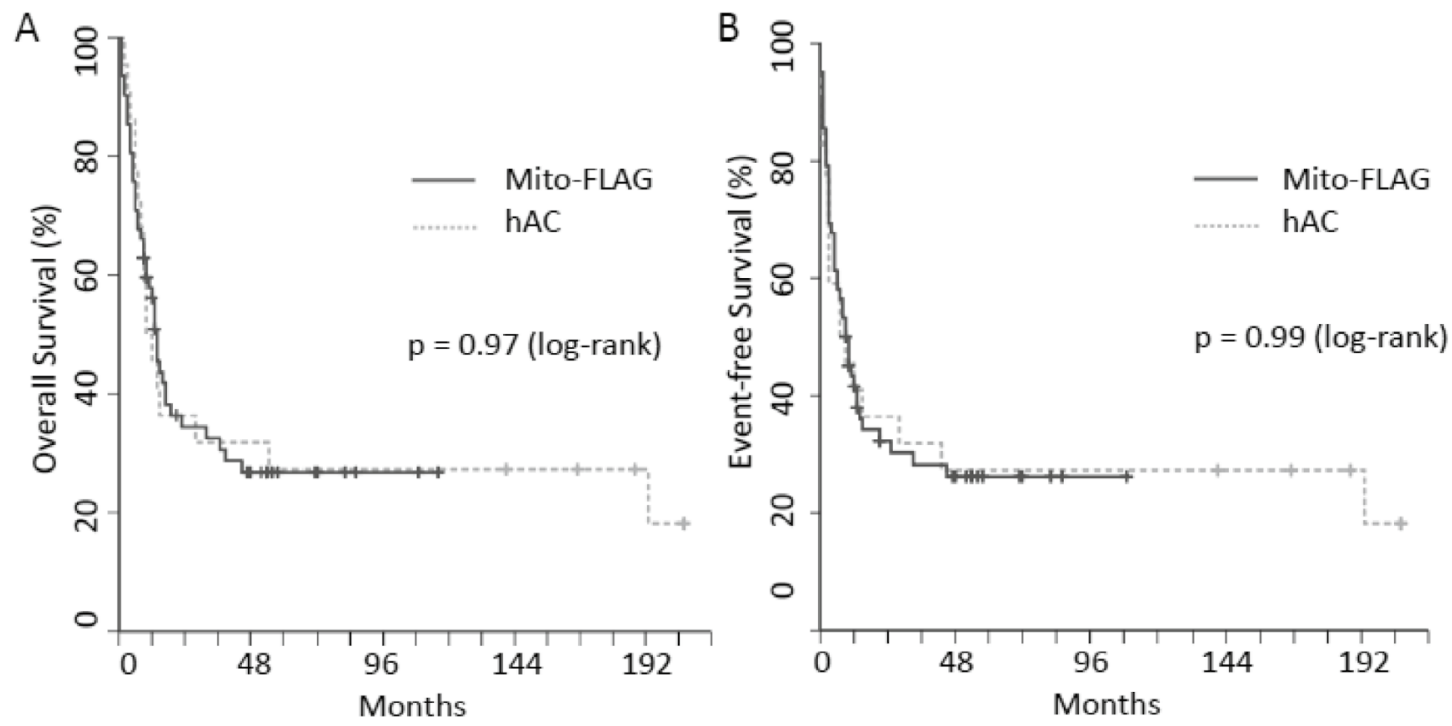

Abbreviations: OS, overall survival; EFS, event-free survival

Fig. 4 OS (A) and EFS (B) of Mito-FLAG and hAC cohort

were all treated at the same center. Patients who received both protocols due to refractoriness to one or the other were excluded. This resulted in the exclusion of one patient of the hAC- and four patients of the Mito-FLAG cohort, respectively. Furthermore, patients who received iAC (intermediate-dose cytarabine and cylcophosphamide) were also excluded since cytarabine doses of iAC are not comparable to cytarabine doses used within the Mito-FLAG regimen. With regard to the limitations of comparing those two cohorts, no statistically significant differences were seen between both salvage regimes (CR rates 54.8\% Mito-FLAG and 54.5\% hAC, respectively).

Refractory versus relapsed AML was the only parameter carrying an impact of achieving remission at the time of Mito-FLAG therapy. While refractory disease was associated with higher probability of $\mathrm{CR}$, this might be in part explained by the fact that 8 of 30 patients who were treated for relapsed AML already underwent alloHSCT prior to Mito-FLAG which harbors a worse prognosis per se. Also, 15 of 36 patients who were treated due to "primary refractory" disease showed in fact PR. Although the definition of refractory disease is not well defined and differs in clinical practice and trials, failure to attain CR to intensive induction therapy is recognized as "primary refractory" (Döhner et al. 2017). A third explanation is that patients not showing complete blast clearance at day 15 bone marrow examination post-induction chemotherapy were considered as not optimal responding and received Mito-FLAG. Out of 16 patients who belong in this category, several patients had shown blast reduction without complete clearance and therefore were treated with Mito-FLAG salvage regimen.

The 30-day and 60-day mortality was $4.5 \%(n=3)$ and $7.6 \%(n=5)$ following first cycle of Mito-FLAG with four patients dying of infectious complications and another patient died of liver failure.

Febrile neutropenia, infectious complications, mucositis and moderate increase in liver enzymes accounted for the majority of adverse events. When comparing with a large analysis of FLAG-Ida salvage therapy, 30- and 60-day mortality was $9.1 \%$ and $15.9 \%$, respectively (Westhus et al. 2019). Owing to the improvements in supportive care especially antimicrobial prophylaxis Mito-FLAG salvage regimen is a feasible and well-tolerated chemotherapy protocol.

With a median follow-up of 54 months, survival analyses demonstrated a median OS of 13 months with a 1-year, 3 -year and 5-year OS of 54.2\%, 30\% and 25\%, respectively. Importantly, $75.8 \%(n=50)$ patients subsequently underwent alloHSCT. The importance of alloHSCT as an essential cornerstone for curative intended treatment is underlined by the fact that all patients that are alive at data cutoff $(n=19)$ were transplanted.

Of note, by log-rank-test no statistical difference was seen when survival analyses of the Mito-FLAG cohort were compared to the hAC cohort treated at the same center. Mito-FLAG patients were notably older than hAC patients. Considering statistical limitations by comparing those two cohorts, it should be pointed out that both salvage regimens 
represent well tolerated and efficacious treatment options for $r / r$ AML.

To date, new treatment strategies for distinct molecular subgroups are available. Gilteritinib, an FLT3-inhibitor led to higher percentages of patients with responses and longer survival than salvage chemotherapy among patients with $\mathrm{r} / \mathrm{r}$ FLT3-mutated AML. In a non-randomized trial the addition of the Bcl-2 inhibitor venetoclax to the FLAG-Ida regimen improved historical outcomes of FLAG-Ida alone, especially when applied as first salvage (DiNardo et al. 2021).

In conclusion, Mito-FLAG therapy is a well-tolerated salvage regimen leading to high response rates in $r / r$ AML. AlloHSCT remains essential as consolidation treatment for long-term disease-free survival in r/r AML patients.

Supplementary Information The online version contains supplementary material available at https://doi.org/10.1007/s00432-021-03821-1.

Funding Open Access funding enabled and organized by Projekt DEAL. No funding.

Availability of data and materials Date are available from the patient records of University Hospital Jena.

\section{Declarations}

Conflict of interest The authors declare no conflict of interest.

Consent to participate Informed consent of patients for participation was obtained when registered for either the OSHO or SAL registry (see above).

Consent to publish Consent of patients to publish was obtained when registered for either the OSHO or SAL registry (see above).

Ethics approval This data analysis was approved by the local Ethics committee of the University Hospital Jena, Germany (Ethical numbers 4871-07/16 for "retrospective evaluation of therapy response and survival in patients with AML" and 3967-12/13 for SAL registry).

Open Access This article is licensed under a Creative Commons Attribution 4.0 International License, which permits use, sharing, adaptation, distribution and reproduction in any medium or format, as long as you give appropriate credit to the original author(s) and the source, provide a link to the Creative Commons licence, and indicate if changes were made. The images or other third party material in this article are included in the article's Creative Commons licence, unless indicated otherwise in a credit line to the material. If material is not included in the article's Creative Commons licence and your intended use is not permitted by statutory regulation or exceeds the permitted use, you will need to obtain permission directly from the copyright holder. To view a copy of this licence, visit http://creativecommons.org/licenses/by/4.0/.

\section{References}

Büchner T, Schlenk RF, Schaich M et al (2012) Acute Myeloid Leukemia (AML): different treatment strategies versus a common standard arm-combined prospective analysis by the German AML Intergroup. J Clin Oncol 30(29):3604-3610

Burnett AK, Russell NH, Hills RK et al (2013) Optimization of chemotherapy for younger patients with acute myeloid leukemia: results of the medical research council AML15 trial. J Clin Oncol 31(27):3360-3368

Burnett AK, Russell NH, Hills RK et al (2015) A randomized comparison of daunorubicin $90 \mathrm{mg} / \mathrm{m}^{2}$ vs $60 \mathrm{mg} / \mathrm{m}^{2}$ in AML induction: results from the UK NCRI AML17 trial in 1206 patients. Blood 125(25):3878-3885

Casper J, Holowiecki J, Trenschel R et al (2012) Allogeneic hematopoietic SCT in patients with AML following treosulfan/fludarabine conditioning. Bone Marrow Transplant 47(9):1171-1177

DiNardo CD, Lachowiez CA, Takahashi K et al (2021) Venetoclax combined with FLAG-IDA induction and consolidation in newly diagnosed and relapsed or refractory acute myeloid leukemia. J Clin Oncol 39:2768-2778

Döhner H, Weisdorf DJ, Bloomfield CD (2015) Acute myeloid leukemia. N Engl J Med 373(12):1136-1152

Döhner H, Estey E, Grimwade D et al (2017) Diagnosis and management of AML in adults: 2017 ELN recommendations from an international expert panel. Blood 129(4):424-447

Dombret H, Gardin C (2016) An update of current treatments for adult acute myeloid leukemia. Blood 127(1):53-61

Estey EH (2020) Acute myeloid leukemia: 2021 update on risk-stratification and management. Am J Hematol 95(11):1368-1398

Fernandez HF, Sun Z, Yao X et al (2009) Anthracycline dose intensification in acute myeloid leukemia. N Engl J Med 361(13):1249-1259

Forman SJ, Rowe JM (2013) The myth of the second remission of acute leukemia in the adult. Blood 121(7):1077-1082

Hänel M, Friedrichsen K, Hänel A et al (2001) Mito-flag as salvage therapy for relapsed and refractory acute myeloid leukemia. Onkologie 24(4):356-360

Heuser M, Ofran Y, Boissel N et al (2020) Acute myeloid leukaemia in adult patients: ESMO Clinical Practice Guidelines for diagnosis, treatment and follow-up. Ann Oncol 31(6):697-712

Jethava YS, Sica S, Savani B et al (2017) Conditioning regimens for allogeneic hematopoietic stem cell transplants in acute myeloid leukemia. Bone Marrow Transplant 52(11):1504-1511

Kahl C, Krahl R, Becker C et al (2016) Long-term follow-up of the AML97 study for patients aged 60 years and above with acute myeloid leukaemia: a study of the East German Haematology and Oncology Study Group (OSHO). J Cancer Res Clin Oncol 142(1):305-315

Kantarjian H, Kadia T, DiNardo C et al (2021) Acute myeloid leukemia: current progress and future directions. Blood Cancer $\mathbf{J}$ 11(2):41

Kell J (2006) Treatment of relapsed acute myeloid leukaemia. Rev Recent Clin Trials 1(2):103-111

Kröger N, Bornhäuser M, Ehninger G et al (2003) Allogeneic stem cell transplantation after a fludarabine/busulfan-based reduced-intensity conditioning in patients with myelodysplastic syndrome or secondary acute myeloid leukemia. Ann Hematol 82(6):336-342

Luo S, Cai F, Jiang L et al (2013) Clinical study of Mito-FLAG regimen in treatment of relapsed acute myeloid leukemia. Exp Ther Med 5(3):982-986

McGowan-Jordan J, Hastings R, Moore S (2021) Re: International System for Human Cytogenetic or Cytogenomic Nomenclature (ISCN): some thoughts, by T. Liehr. Cytogenet Genome Res. https://doi.org/10.1159/000516655

Megías-Vericat JE, Martínez-Cuadrón D, Sanz M, Montesinos P (2018) Salvage regimens using conventional chemotherapy agents for relapsed/refractory adult AML patients: a systematic literature review. Ann Hematol 97(7):1115-1153 
Parker JE, Pagliuca A, Mijovic A et al (1997) Fludarabine, cytarabine, G-CSF and idarubicin (FLAG-IDA) for the treatment of poor-risk myelodysplastic syndromes and acute myeloid leukaemia. Br J Haematol 99(4):939-944

Perl AE, Martinelli G, Cortes JE et al (2019) Gilteritinib or chemotherapy for relapsed or refractory FLT3-mutated AML. N Engl J Med 381(18):1728-1740

Roboz GJ, Rosenblat T, Arellano M et al (2014) International randomized phase III study of elacytarabine versus investigator choice in patients with relapsed/refractory acute myeloid leukemia. J Clin Oncol 32(18):1919-1926

Röllig C, Kramer M, Schliemann C et al (2020) Does time from diagnosis to treatment affect the prognosis of patients with newly diagnosed acute myeloid leukemia? Blood 136(7):823-830

Schmid C, Schleuning M, Schwerdtfeger R et al (2006) Long-term survival in refractory acute myeloid leukemia after sequential treatment with chemotherapy and reduced-intensity conditioning for allogeneic stem cell transplantation. Blood 108(3):1092-1099

Schnetzke U, Fix P, Spies-Weisshart B et al (2014) Efficacy and feasibility of cyclophosphamide combined with intermediate- dose or high-dose cytarabine for relapsed and refractory acute myeloid leukemia (AML). J Cancer Res Clin Oncol 140(8):1391-1397

Scholl S, Krause C, Loncarevic IF et al (2005) Specific detection of Flt3 point mutations by highly sensitive real-time polymerase chain reaction in acute myeloid leukemia. J Lab Clin Med 145(6):295-304

Scholl S, Theuer C, Scheble V et al (2008) Clinical impact of nucleophosmin mutations and Flt3 internal tandem duplications in patients older than $60 \mathrm{yr}$ with acute myeloid leukaemia. Eur J Haematol 80(3):208-215
Short NJ, Rytting ME, Cortes JE (2018) Acute myeloid leukaemia. Lancet 392(10147):593-606

Spadea A, Petti MC, Fazi P et al (1993) Mitoxantrone, etoposide and intermediate-dose Ara-C (MEC): an effective regimen for poor risk acute myeloid leukemia. Leukemia 7(4):549-552

Steinmetz HT, Schulz A, Staib P et al (1999) Phase-II trial of idarubicin, fludarabine, cytosine arabinoside, and filgrastim (IdaFLAG) for treatment of refractory, relapsed, and secondary AML. Ann Hematol 78(9):418-425

Thiel A, Schetelig J, Pönisch W et al (2015) Mito-FLAG with Ara-C as bolus versus continuous infusion in recurrent or refractory AML-long-term results of a prospective randomized intergroup study of the East German Study Group Hematology/Oncology (OSHO) and the Study Alliance Leukemia (SAL). Ann Oncol 26(7):1434-1440

Thol F, Schlenk RF, Heuser M, Ganser A (2015) How I treat refractory and early relapsed acute myeloid leukemia. Blood 126(3):319-327

Westhus J, Noppeney R, Dührsen U, Hanoun M (2019) FLAG salvage therapy combined with idarubicin in relapsed/refractory acute myeloid leukemia. Leuk Lymphoma 60(4):1014-1022

Wiernik PH, Banks PL, Case DC Jr et al (1992) Cytarabine plus idarubicin or daunorubicin as induction and consolidation therapy for previously untreated adult patients with acute myeloid leukemia. Blood 79(2):313-319

Publisher's Note Springer Nature remains neutral with regard to jurisdictional claims in published maps and institutional affiliations. 